Disclosure of Interests: Carmen Álvarez-Reguera: None declared, Jorge Javier Gaitán-Valdizán: None declared, Raúl Fernández-Ramón: None declared, Rosalía Demetrio-Pablo: None declared, José Luis Martín-Varillas: None declared, Lara Sanchez-Bilbao: None declared, David Martínez-López: None declared, Iñigo González-Mazón: None declared, Miguel Á. González-Gay Speakers bureau: Abbvie, Pfizer, Roche, Sanofi and MSD., Grant/research support from: Abbvie, MSD, Janssen and Roche., Ricardo Blanco Speakers bureau: Abbvie, Pfizer, Roche, Bristol-Myer, Janssen, Lilly and MSD., Grant/research support from: Abbvie, MSD and Roche.

DOI: 10.1136/annrheumdis-2021-eular.1930

\section{POS0741 HISTOPATHOLOGIC PATTERNS OF LUPUS NEPHRITIS PREDICT THE RISKS OF MORTALITY A SINGLE-CENTER RETROSPECTIVE STUDY}

$\underline{\text { Y. W. Liao }}^{1}$, W. T. Hung ${ }^{1}$, Y. M. Chen ${ }^{1}$, C. W. Hsieh ${ }^{1}$, T. Y. Hsieh ${ }^{1}$, Y. H. Chen ${ }^{1}$, W. N. Huang'. ' Taichung Veterans General Hospital, Division of Allergy, Immunology and Rheumatology, Taichung, Taiwan, Republic of China

Background: Lupus nephritis is a significant complication of systemic lupus erythematosus and is associated with increased risks of end-stage kidney disease and mortality.

Objectives: The retrospective observational study aims to investigate which component of the National Institutes of Health activity and chronic indices of lupus nephritis can predict mortality.

Methods: We identified 528 SLE patients with biopsy-proven lupus nephritis between 2006 and 2019. Two patients with class VI lupus nephritis were excluded, and a total of 526 patients were analyzed. Serum creatinine, urine protein-to-creatinine ratio (UPCR), and serologic markers for SLE disease activity were measured at the time of the renal biopsy. The histopathologic findings of renal biopsies were classified by utilizing the International Society of Nephrology/ Renal Pathology Society (ISN/RPS) classification.

Results: Among 526 patients enrolled, 64 expired, and 44 were female $(68.8 \%, p=0.004)$. Class IV $( \pm V)$ comprised the most $(n=39,60.9 \%)$, followed by class $V(n=18,29.7 \%)$. Lower eGFR was observed in the death group, compared with the survival group (median: 24.7 vs. $80.5, p<0.001$ ). There were no significant differences in UPCR and serologic markers for SLE (dsDNA, C3, and C4). Total scores of chronicity index and the scores for each index were higher in the death group. Interestingly, although total scores of activity index in death and survival groups did not differ significantly, the scores for cellular crescents tended to be higher in the death group $(1.38 \pm 1.77$ vs. $0.72 \pm 1.24$, $\mathrm{p}=0.002$ )

In the univariable analysis, age, male sex, eGFR, activity index scores, cellular crescents, chronicity index scores, and all $\mathrm{Cl}$ components (global obsolete glomeruli, tubular atrophy, interstitial fibrosis, fibrous crescents) and tubulointerstitial nephritis were significantly associated with an increased risk of death. When patient characteristics and $\mathrm{NIH}$ activity/ chronicity indices were jointly examined in a multivariable analysis, fibrous crescents were significantly associated with increased risk of death in females (HR 5.23 [95\% Cl: 1.51, 18.09]) (Table 1). In males, the risks of death increased with cellular crescents (HR 1.73 [95\% $\mathrm{Cl}: 1.10,2.73])$ but decreased with global obsolete glomeruli $(\mathrm{HR} 0.12[95 \% \mathrm{Cl}$ : $0.02,0.91])$.

Conclusion: In this single-center observational study, fibrous crescents in females and cellular crescents in males were significantly associated with increased risks of mortality.

REFERENCES:

[1] Doria A, laccarino L, Ghirardello A, et al. Long-term prognosis and causes of death in systemic lupus erythematosus. Am J Med 2006; 119: 700-706.
[2] Faurschou M, Starklint H, Halberg P, Jacobsen S. Prognostic factors in lupus nephritis: diagnostic and therapeutic delay increases the risk of terminal renal failure. J Rheumatol. 2006;33(8):1563-1569.

[3] Chen YM, Hung WT, Liao YW, et al. Combination immunosuppressant therapy and lupus nephritis outcome: a hospital-based study. Lupus. 2019;28(5):658-666.

Disclosure of Interests: None declared

DOI: 10.1136/annrheumdis-2021-eular.2006

\section{\begin{tabular}{|l|l}
\hline POS0742 & SCREENING AND BIOINFORMATICS ANALYSIS \\
\hline
\end{tabular} OF HUB GENES AND PATHWAYS FOR PRIMARY SJÖGREN'S SYNDROME BASED ON GEO DATABASE}

T. Kong ${ }^{1}$, S. X. Zhang ${ }^{2,3,4}$, S. Song ${ }^{2,3,4}$, X. Sun ${ }^{1}$, C. Zheng ${ }^{1}$, S. Feng ${ }^{5}$, L. Cheng ${ }^{1}$, G. Shi ${ }^{6}$, X. Li ${ }^{2,3,4}$, P. F. He ${ }^{7}$, Q. Yu ${ }^{1,7} .{ }^{1}$ Shanxi Medical University, School of Management, Taiyuan, China; ${ }^{2}$ The Second Hospital of Shanxi Medical University, Department of Rheumatology, Taiyuan, China; ${ }^{3}$ Shanxi Li Xiaofeng Medical Groups, Department of Rheumatology, Taiyuan, China; ${ }^{4}$ Ministry of Education, Key Laboratory of Cellular Physiology at Shanxi Medical University, Taiyuan, China; ${ }^{5}$ Shanxi Medical University, School of Humanities and Social Sciences, Taiyuan, China; ${ }^{6}$ Shanxi Medical University, Basic Medical College, Taiyuan, China; ${ }^{7}$ Shanxi Medical University, Institute of Medical Data Sciences, Taiyuan, China

Background: Primary Sjögren's syndrome (pSS) is an autoimmune disease that featured as lymphoplasmacytic infiltration of the exocrine glands leading to sicca symptoms ${ }^{1}$. However, its underlying molecular mechanisms remain elusive.

Objectives: This study aims to identify differentially expressed genes (DEGs) and pathways associated with the progression of pSS using bioinformatics analysis and explore its pathogenesis.

Methods: The pSS-associated gene chip data set GSE66795 was obtained from the Gene Expression Omnibus (GEO) database, which included 131 cases of fully-phenotyped pSS patients' whole blood samples and 29 cases of control samples. DEGs were screened Using R software. Online tool Metascape ${ }^{2}$ was used to make Gene Ontology (GO) and KEGG pathway enrichment. The PPI network was performed using String database. Hub genes were identified by Cytoscape.

Results: A total of 108 DEGs were captured, including 101 up-regulated genes and 7 down-regulated genes. GO enrichment showed that these DEGs were primarily enriched in defense response to virus, response to interferon-gamma, regulation of innate immune response, response to interferon-beta, double-stranded RNA binding, response to interferon-alpha. KEGG pathway enrichment analysis showed these DEGs were principally enriched in Influenza A, RIG-I-like receptor signaling pathway, necroptosis, Staphylococcus aureus infection. Finally, 9 hub genes (STAT1, IRF7, OAS2, GBP1, OAS1, IFIT3, IFIH1, OAS3, DDX60) had highest degree value.

Conclusion: The findings identified molecular mechanisms and the key hub genes that may involve in the occurrence and development of pSS.

REFERENCES:

[1] Francois $\mathrm{H}$, Mariette $\mathrm{X}$. Renal involvement in primary Sjogren syndrome. Nat Rev Nephrol 2016;12(2):82-93. doi: 10.1038/nrneph.2015.174 [published Online First: 2015/11/17].

[2] Zhou Y, Zhou B, Pache L, et al. Metascape provides a biologist-oriented resource for the analysis of systems-level datasets. Nat Commun 2019;10(1):1523. doi: 10.1038/s41467-019-09234-6 [published Online First: 2019/04/05].

Table 1. Logistic regression of predictors for mortality in patients with lupus nephritis

\begin{tabular}{|c|c|c|c|c|c|c|c|c|c|}
\hline & \multicolumn{3}{|c|}{ Univariable } & \multicolumn{4}{|c|}{ Multivariable (Female) } & \multicolumn{2}{|c|}{ Multivariable (Male) } \\
\hline & $\mathrm{HR}$ & $95 \% \mathrm{Cl}$ & $p$ value & $\mathrm{HR}$ & $95 \% \mathrm{Cl}$ & $p$ value & $\mathrm{HR}$ & $95 \% \mathrm{Cl}$ & $p$ value \\
\hline Age & 1.03 & $(1.01-1.05)$ & 0.002 & 1.02 & $(0.98-1.07)$ & 0.267 & 1.01 & $(0.95-1.08)$ & 0.670 \\
\hline Male sex & 2.10 & $(1.23-3.55)$ & 0.006 & & & & & & \\
\hline UPCR & 1.02 & $(0.95-1.09)$ & 0.616 & & & & & & \\
\hline eGFR & 0.97 & $(0.96-0.99)$ & $<0.001$ & 0.99 & $(0.96-1.00)$ & 0.183 & 0.98 & $(0.96-1.00)$ & 0.086 \\
\hline Activity Index & 1.06 & $(1.01-1.11)$ & 0.027 & & & & & & \\
\hline Cellular crescents & 1.29 & $(1.12-1.50)$ & $<0.001$ & 1.03 & $(0.63-1.67)$ & 0.917 & 1.73 & $(1.10-2.73)$ & 0.017 \\
\hline Chronicity Index & 1.16 & $(1.07-1.26)$ & $<0.001$ & & & & & & \\
\hline global obsolete glomeruli & 1.37 & $(1.08-1.76)$ & 0.011 & 1.24 & $(0.55-2.77)$ & 0.606 & 0.12 & $(0.02-0.91)$ & 0.040 \\
\hline Tubular atrophy & 1.65 & $(1.28-2.13)$ & $<0.001$ & 0.41 & $(0.06-2.82)$ & 0.362 & 4.77 & $(0.30-75.32)$ & 0.267 \\
\hline Interstitial fibrosis & 1.71 & $(1.32-2.23)$ & $<0.001$ & 3.70 & (0.52-26.24) & 0.191 & 1.37 & $(0.07-27.40)$ & 0.837 \\
\hline Fibrous crescents & 2.38 & $(1.40-4.03)$ & 0.001 & 5.23 & $(1.51-18.09)$ & 0.009 & 0.00 & (0-extremely large) & 0.989 \\
\hline Tubulointerstitial nephritis & 1.70 & $(1.03-2.80)$ & 0.037 & & & & & & \\
\hline
\end{tabular}

